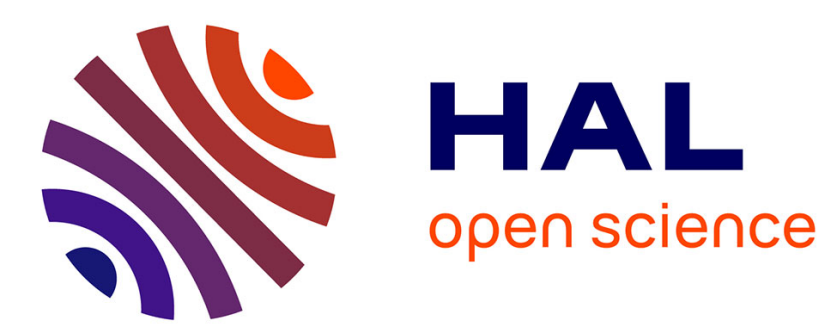

\title{
Nonquadratic Local Risk-Minimization for Hedging Contingent Claims in Incomplete Markets
}

\author{
Frédéric Abergel, Nicolas Millot
}

\section{To cite this version:}

Frédéric Abergel, Nicolas Millot. Nonquadratic Local Risk-Minimization for Hedging Contingent Claims in Incomplete Markets. SIAM Journal on Financial Mathematics, 2011, 2 (1), pp. 342-356. 10.1137/100803079 . hal-00620843

\section{HAL Id: hal-00620843 https://hal.science/hal-00620843}

Submitted on 8 Sep 2011

HAL is a multi-disciplinary open access archive for the deposit and dissemination of scientific research documents, whether they are published or not. The documents may come from teaching and research institutions in France or abroad, or from public or private research centers.
L'archive ouverte pluridisciplinaire HAL, est destinée au dépôt et à la diffusion de documents scientifiques de niveau recherche, publiés ou non, émanant des établissements d'enseignement et de recherche français ou étrangers, des laboratoires publics ou privés. 


\title{
Nonquadratic Local Risk-Minimization for Hedging Contingent Claims in Incomplete Markets*
}

\author{
Frédéric Abergel ${ }^{\dagger}$ and Nicolas Millot ${ }^{\dagger}$
}

\begin{abstract}
We introduce a new criterion to perform hedging of contingent claims in incomplete markets. Our approach is close to the one proposed by Schweizer [Stochastic Process. Appl., 37 (1991), pp. 339363] in that it uses the concept of locally risk-minimizing strategies. But we aim at being more general by defining the local risk as a general, nonnecessarily quadratic, convex function of the local cost process. We derive the corresponding optimal strategies and value function in both discrete and continuous time settings. Finally we give an application of our hedging method in the stochastic volatility case as well as in the jump diffusion case. We work with a single traded asset, but our approach may be generalized to deal with claims depending on multiple assets.
\end{abstract}

Key words. hedging, incomplete markets, local risk-minimization, derivatives

AMS subject classifications. 91G20, 91G80, 49J20, 93E20

DOI. $10.1137 / 100803079$

1. Motivations. Using a quadratic function to assess the risk associated with some cost process means that we will equally penalize the profits and losses generated by our trading strategy. Though this approach leads to some neat mathematical developments (see the important literature on quadratic local risk-minimization or on mean-variance hedging such as Föllmer and Sondermann [8], Föllmer and Schweizer [6], [7], or Heath, Platen, and Schweizer [9]) it probably does not perfectly reflect the way market practitioners assess their risk. In order to overcome this, we shall introduce a risk function $f,{ }^{1} f \in \mathcal{C}^{3}(\mathbb{R}, \mathbb{R})$ with $f(0)=f^{\prime}(0)=$ 0 , strictly convex and with $\lim _{|x| \rightarrow \infty} f(x)=+\infty$, that will typically allow putting more weight on losses than on profits.

2. Discrete time setting. We first place ourselves in a multiperiod model where the evolution of the stock price is given by a stochastic process $S_{k}(k=0, \ldots, T)$ on some probability space $(\Omega, \mathcal{F}, P)$. Let $\mathcal{F}_{k}$ denote the $\sigma$-field of events which are observable up to and including time $k$. We assume that $S_{k}$ is adapted and square-integrable. In order to avoid complicated notations, we work with zero interest rates, but this is not a loss of generality, as it basically amounts to having discounted stock prices with the existence of a "nonrisky" asset.

A contingent claim is described by a square-integrable random variable $H \in L^{2}(P)$ that will be assumed of the form $H=\delta^{H} S_{T}+\beta^{H}$ with $\delta^{H}$ and $\beta^{H}$ being $\mathcal{F}_{T}$-measurable ran-

\footnotetext{
${ }^{*}$ Received by the editors July 21, 2010; accepted for publication (in revised form) February 5, 2011; published electronically May 24, 2011.

http://www.siam.org/journals/sifin/2/80307.html

${ }^{\dagger}$ Chaire de Finance Quantitative, Laboratoire de Mathématiques Appliquées aux Systèmes, École Centrale Paris, 92290 Châtenay-Malabry, France (frederic.abergel@ecp.fr, nicolas.millot@ecp.fr).

${ }^{1}$ We will further assume that $f$ has at most quadratic growth in order to circumvent integrability conditions by working in $L^{2}(P)$, where $P$ is one chosen probability measure.
} 
dom variables. Thus we are considering European-type options that can have a cash settled component and a deliverable one.

For example, a call option with strike $K$ would correspond to $H=\left(S_{T}-K\right)^{+}=1_{S_{T} \geq K} S_{T}-$ $K 1_{S_{T} \geq K}$, so that $\delta^{H}=1_{S_{T} \geq K}$ and $\beta^{H}=-K 1_{S_{T} \geq K}$.

2.1. Trading strategies. A trading strategy $\Phi$ is given by two stochastic processes $\delta_{k}(k=$ $0, \ldots, T)$ and $\beta_{k}(k=0, \ldots, T) . \delta_{k}$ is the amount of stock held in period $k,\left(=\left[t_{k}, t_{k+1}\right)\right)$ and has to be fixed at the beginning of that period; i.e., we assume that $\delta_{k}$ is $\mathcal{F}_{k}$-measurable $(k=0, \ldots, T)$.

The amount $\beta_{k}$ held in the market account in period $k$ is fixed at the beginning of this period too; i.e., we assume that $\beta_{k}$ is $\mathcal{F}_{k}$-measurable $(k=0, \ldots, T)$.

We further assume that both $\delta$ and $\beta$ are in $L^{2}(P)$.

The reader familiar with the local risk-minimization framework will already notice the difference from the usual setting, where typically only the cash component $\beta$ of the strategy $\Phi$ is allowed to be adapted. The relaxation of the predictability of the strategy in both its components allows us to consider both types of options (cash settled and deliverable) and will allow for a generalization to the case when there are liquidity costs.

For such a trading strategy, the theoretical value of the portfolio at time $k$ is given by

$$
V_{k}=\delta_{k} S_{k}+\beta_{k}(k=1, \ldots, T),
$$

and so it is its value just after applying the strategy. We will denote by $H$-admissible trading strategies which satisfy that each $V_{k}$ is square-integrable and such that the contingent claim $H$ is produced in the end; i.e., we require $V_{T}=H$, which can always be met with our measurability requirements upon taking $\delta_{T}=\delta_{T}^{H}$ and $\beta_{T}=\beta_{T}^{H}$.

2.2. Costs and risk processes. The costs $\Delta C_{k}$ incurred at time $t_{k}, k>0$, from following strategy $\Phi$, so from changing the stock amount that we hold from $\delta_{k-1}$ to $\delta_{k}$ and from changing the amount invested in the money market account from $\beta_{k-1}$ to $\beta_{k}$, are given in the absence of transaction $\operatorname{costs}^{2}$ by

$$
\begin{aligned}
\Delta C_{k}(\Phi) & =\left(\delta_{k}-\delta_{k-1}\right) S_{k}+\left(\beta_{k}-\beta_{k-1}\right) \quad \forall k \in\{k=1, \ldots, T\} \\
\Leftrightarrow \Delta C_{k}(\Phi) & =V_{k}-V_{k-1}-\delta_{k-1}\left(S_{k}-S_{k-1}\right) \quad \forall k \in\{k=1, \ldots, T\} .
\end{aligned}
$$

We then define the local risk $\Delta R_{k}$ at time $k$ associated with the costs incurred at time $k+1$. It is

$$
\Delta R_{k}(\Phi)=\mathbb{E}\left(f\left(\Delta C_{k+1}\right) \mid \mathcal{F}_{k}\right)
$$

or with obvious notation

$$
\Delta R_{k}(\Phi)=\mathbb{E}_{k}\left(f\left(\Delta C_{k+1}\right)\right) .
$$

Our objective is now to find those trading strategies that will sequentially minimize the risk process. We make this statement precise in the next section.

\footnotetext{
${ }^{2}$ Fully incorporating transaction costs would introduce a dependence on the sign of the readjustment as well as a nonlinear dependence on the quantity; see [1] for the case where there are only liquidity costs.
}

Copyright $\odot$ by SIAM. Unauthorized reproduction of this article is prohibited. 
2.3. Locally risk-minimizing strategies. The sequential minimization program runs backward in time; i.e., given $\left(\delta_{k+1}^{*}, \delta_{k+2}^{*}, \ldots, \delta_{T}^{*}\right)$ and $\left(\beta_{k+1}^{*}, \beta_{k+2}^{*}, \ldots, \beta_{T}^{*}\right)$ (or equivalently $\left.\left(V_{k+1}^{*}, V_{k+2}^{*}, \ldots, V_{T}^{*}\right)\right)$, we look for $\delta_{k}^{*}$ and $\beta_{k}^{*}$ (or $\left.V_{k}^{*}\right)$ such that $\Delta R_{k}$ is minimized.

Problem (*). Given a contingent claim $H$, find a $\Phi^{*}, H$-admissible strategy such that

$$
\begin{aligned}
\forall k \in(0, \ldots, T-1), \Delta R_{k}(\Phi) & \geq \Delta R_{k}\left(\Phi^{*}\right) \quad \forall \Phi H \text {-admissible, } \\
\text { with } \delta_{k+1} & =\delta_{k+1}^{*} \quad \text { and } \quad \beta_{k+1}=\beta_{k+1}^{*} .
\end{aligned}
$$

Given the conditions imposed on $f, S_{k}$, and $\beta_{k}$ (or $V_{k}$ ), we have the existence and uniqueness of the optimal strategy, and it is characterized through the first-order optimality equations

$$
\left\{\begin{aligned}
\mathbb{E}_{k}\left(f^{\prime}\left(\Delta C_{k+1}\left(\Phi^{*}\right)\right)\right) & =0, \\
\mathbb{E}_{k}\left(f^{\prime}\left(\Delta C_{k+1}\left(\Phi^{*}\right)\right) \Delta S_{k+1}\right) & =0 .
\end{aligned}\right.
$$

The above equations will prove useful for further characterizing optimal strategies, particularly in continuous time, but it is important to notice that the existence and uniqueness of the solution of problem $\left.{ }^{*}\right)$ hold even though $f$ is not regular: all that is required is that it be strictly convex with suitable bounds as prescribed in the introduction. The characterizing set of equations (1), on the other hand, holds only when $f$ is differentiable.

Theorem 1. Problem $(*)$ has a unique solution $\Phi^{*}$ whose components $\delta^{*}$ and $\beta^{*}$ solve the set of equations (1).

Proof. Let $h(x, y, \omega) \equiv E_{k}(f((U-x) S+(V-y)))(\omega)$ with $U, V$, and $S \in \mathcal{L}^{2}(P)$. First, we observe that for a fixed $\omega, h$ is a convex function of $x$ and $y$ so that it has a global minimum $\left(x^{*}, y^{*}\right)$ if and only if $\left(x^{*}, y^{*}\right)$ is a critical point of $h$, i.e., $\nabla h\left(x^{*}, y^{*}\right)=0$. Second, we have $\lim _{\|(x, y)\| \rightarrow \infty} h(x, y, \omega)=+\infty$ so that $h$ being continuous it has a global minimum $P$-a.s. Finally, we show that $\left(x^{*}, y^{*}\right)$ is $\mathcal{F}_{k}$-measurable: letting $D_{n}=\left\{j 2^{-n} \mid j \in \mathbb{Z}\right\}$ be the set of dyadic rational of order $n$, we define

$$
\left(x_{n}(\omega), y_{n}(\omega)\right)=\inf \left\{(x, y) \in D_{n} \times D_{n}, h(x, y, \omega) \leq h\left(x^{\prime}, y^{\prime}, \omega\right) \forall\left(x^{\prime}, y^{\prime}\right) \in D_{n} \times D_{n}\right\} .
$$

Since $\omega \mapsto h(x, y, \omega)$ is $\mathcal{F}_{k}$-measurable, $\left(x_{n}, y_{n}\right)$ is also $\mathcal{F}_{k}$-measurable. As $\left(x_{n}, y_{n}\right)$ is bounded in $n$ P-a.e. and $h$ is continuous in $(x, y),(\tilde{x}, \tilde{y})=\liminf _{n \rightarrow \infty}\left(x_{n}, y_{n}\right)$ is a $\mathcal{F}_{k^{-}}$ measurable minimizer of $h$ and by uniqueness it is equal to $\left(x^{*}, y^{*}\right)$.

The set of equations (1) is equivalent to the property that the process $\left(C_{k}^{f}\right)_{k}$ with $C_{k}^{f}=$ $\sum_{i=0}^{k} f^{\prime}\left(\Delta C_{i+1}\right)$-which we will refer to as the $f$-cost process - is a martingale strongly orthogonal to $S_{k}$. This property, which we name pseudo-optimality as in Schweizer [12], will be the main ingredient of the extensions to the continuous time setting.

3. Continuous time setting. Now let $(\Omega, \mathcal{F}, P)$ be a probability space with a filtration $\left(\mathcal{F}_{t}\right)_{0 \leq t \leq T}$ satisfying the usual conditions of right-continuity and completeness. $T \in \mathbb{R}^{+}$denotes a fixed and finite time horizon. Furthermore, we assume that $\mathcal{F}_{0}$ is trivial and that $\mathcal{F}_{T}=\mathcal{F}$. Let $S=\left(S_{t}\right)_{0 \leq t \leq T}$ be a semimartingale with a decomposition

$$
S=S_{0}+M+A
$$

such that $M=\left(M_{t}\right)_{0 \leq t \leq T}$ is a square-integrable martingale with $M_{0}=0$ and $A=\left(A_{t}\right)_{0 \leq t \leq T}$ is a continuous and adapted process of finite variation $|A|$ with $A_{0}=0$. Throughout the article, we use a right-continuous version of $S$.

Copyright $@$ by SIAM. Unauthorized reproduction of this article is prohibited. 
3.1. Trading strategies. A trading strategy $\Phi$ is a pair of processes $\delta=\left(\delta_{t}\right)_{0 \leq t \leq T}, \beta=$ $\left(\beta_{t}\right)_{0 \leq t \leq T}$ satisfying the following conditions:

$$
\left\{\begin{array}{l}
\delta \text { is càdlàg and adapted, } \\
\beta \text { is càdlàg and adapted. }
\end{array}\right.
$$

An option is again described by a square-integrable random variable $H \in L^{2}(P)$, with $H=\delta^{H} S_{T}+\beta^{H}, \delta^{H}$ and $\beta^{H}$ being $\mathcal{F}_{T}$-measurable random variables.

So as to make precise what strategies can be considered, we first need to introduce some classical notations and definitions. Let $\left(X_{t}\right)$ and $\left(Y_{t}\right)$ be two stochastic processes. We will also refer to the portfolio value $V_{t}$ at time $t$ as the quantity $V_{t}=\delta_{t} S_{t}+\beta_{t}$.

If $\mathcal{P}$ is a partition of $[0, T]$ such that

$$
\mathcal{P}=\left\{0=\tau_{0} \leq \tau_{1} \leq \cdots \leq \tau_{n}=T\right\},
$$

then we recall that the quadratic variation process along $\mathcal{P}$ is

$$
[X, X]_{t}^{\mathcal{P}}=\sum_{k=1}^{n}\left(X_{\tau_{k} \wedge t}-X_{\tau_{k-1} \wedge t}\right)^{2} .
$$

And the quadratic covariation process along $\mathcal{P}$ is

$$
[X, Y]_{t}^{\mathcal{P}}=\sum_{k=1}^{n}\left(X_{\tau_{k} \wedge t}-X_{\tau_{k-1} \wedge t}\right)\left(Y_{\tau_{k} \wedge t}-Y_{\tau_{k-1} \wedge t}\right) .
$$

We will work with sequences of Riemann partitions $\mathcal{P}_{n}$ which satisfy $\left|\mathcal{P}_{n}\right| \equiv \sup _{k}\left|\tau_{k}^{n}-\tau_{k-1}^{n}\right|$ tends to 0 as $n \rightarrow \infty$.

Let us finally recall that $X$ is said to have finite quadratic variation on $[0, T]$ if $\lim _{n \rightarrow \infty}[X, X]^{\mathcal{P}_{n}}$ exists in the topology of uniform convergence in probability for any sequence $\mathcal{P}_{n}$ of Riemann partitions.

Now we are in a position to introduce some restrictions on our strategies so that the optimality conditions are well defined. We shall concentrate on strategies which are $H$-admissible in the sense that

$$
\left\{\begin{array}{l}
\delta_{T}=\delta^{H} P \text {-a.s., } \\
\beta_{T}=\beta^{H} P \text {-a.s., } \\
\delta \text { has finite and integrable quadratic variation, } \\
\beta \text { has finite and integrable quadratic variation, } \\
\delta \text { and } \beta \text { have finite and integrable quadratic covariation. }
\end{array}\right.
$$

The justification for considering such strategies will be given in the next section.

3.2. Local risk-minimization and the $f$-cost process. This section is devoted to the two main concepts that will allow us to find optimal strategies and the relationship with them in a particular framework where $S$ is driven by an Itô process. We start by introducing the local risk-minimization criterion and then define the $f$-cost process needed to derive pseudo-optimal strategies.

Copyright (C) by SIAM. Unauthorized reproduction of this article is prohibited. 
Local risk-minimization. In order to extend the idea of local risk-minimization already seen in discrete time to our continuous time framework, we first introduce the concept of small perturbations and then characterize the optimal strategies as the ones that minimize the local risk, at the limit, with respect to these small perturbations.

\section{Small perturbations.}

Definition. A small perturbation is a bounded admissible ${ }^{3}$ strategy $\phi=(\beta, \delta)$ such that $\beta_{T}=0$ and $\delta_{T}=0$.

Local risk along a partition. We start with an $H$-admissible strategy $\Phi$, and we want to study the increase of risk when the strategy is perturbed at some discrete times. To do so, given a partition $\tau$ of $[0, T]$, where $\tau=\left\{0=t_{0}, t_{1}, \ldots, t_{k}=T\right\}$, and a small perturbation $\Delta$, we define a process $r_{f}^{\tau}$ in the following way:

$$
r_{f}^{\tau}[\Phi, \phi](t, \omega)=\sum_{t_{i}, t_{i+1} \in \tau} \frac{\Delta R_{t_{i}}\left(\Phi+\left.\phi\right|_{\left[t_{i}, t_{i+1}()\right.}(\omega)-\Delta R_{t_{i}}(\Phi)(\omega)\right.}{t_{i+1}-t_{i}} 1_{\left[t_{i}, t_{i+1}(\right.}(t)
$$

with $\Delta R_{t_{i}}(\Phi)=\mathbb{E}\left(f\left(\Delta C_{t_{i+1}}\right) \mid \mathcal{F}_{t_{i}}\right)$.

Now we can define the local risk-minimization in the same way as we did for the discrete time setting.

Definition. An $H$-admissible strategy $\Phi$ is called locally risk-minimizing for the option $H$ if for every small perturbation $\phi$ and every increasing sequence of partitions $\left(\tau_{n}\right)_{n \in \mathbb{N}}$ tending to the identity we have

$$
\liminf _{n \rightarrow \infty} r^{\tau_{n}}[\Phi, \phi] \geq 0 \mathcal{P} \text {-a.e. }
$$

As a matter of fact, this definition naturally extends the notion of local minimization of a local risk. However, the associated optimality conditions are not readily derived in the general semimartingale case. In the following section, we will introduce the concept of a pseudo-optimal strategy similar to the discrete time setting. In the case where the asset follows an Itô process, one can show that optimal and pseudo-optimal strategies are the same. This equivalence will be worked out in detail for the particularly relevant situations of stochastic volatility models in the last section of this article.

The $f$-cost process. Now we proceed with defining the process $f$-cost process which will allow us to characterize pseudo-optimal strategies by analogy with discrete time.

Definition. For an $H$-admissible strategy $\Phi$ we define the $f$-cost process $C_{t}(\Phi)$ as the following limit, whenever it exists:

$$
\lim _{n \rightarrow \infty} \sum_{k=1}^{l_{n}} f^{\prime}\left(\beta^{\tau_{k}^{n}}-\beta^{\tau_{k-1}^{n}}+\left(\delta^{\tau_{k}^{n}}-\delta^{\tau_{k-1}^{n}}\right) S^{\tau_{k}^{n}}\right),
$$

where convergence is required in ucp topology, for any sequences $\mathcal{P}_{n}$ of Riemann partitions of $[0, T]$ of length $l_{n}$, and where we used the notation $X^{T}$ for the process stopped at $T$.

\footnotetext{
${ }^{3}$ Admissible means that it satisfies the same regularity requirements as an $H$-admissible strategy with different terminal conditions.
}

Copyright ( $)$ by SIAM. Unauthorized reproduction of this article is prohibited. 
We now focus on an $H$-admissible strategy $\Phi$ and state a theorem relative to the existence of the $f$-cost process. ${ }^{4}$

Theorem 2. The $f$-cost process of an $H$-admissible strategy $\Phi$ is well defined and is given by the following formula:

$$
\begin{aligned}
C_{t}(\Phi)= & f^{\prime \prime}(0)\left(V_{t}-V_{0}-\int_{0+}^{t} \delta_{s-} d S_{s}\right) \\
& +\frac{f^{(3)}(0)}{2}\left([\beta, \beta]_{t}^{c}+2 \int_{0+}^{t} S_{s-} d[\beta, \delta]_{s}^{c}+\int_{0+}^{t} S_{s-}^{2} d[\delta, \delta]_{t}^{c}\right) \\
& +\sum_{0<s \leq t} f^{\prime}\left(\Delta \beta_{s}+\Delta \delta_{s} S_{s}\right)-f^{\prime \prime}(0)\left(\Delta \beta_{s}+\Delta \delta_{s} S_{s}\right)
\end{aligned}
$$

with notation $[X, Y]^{c}$ standing for the continuous part of the (càdlàg) quadratic covariation process.

Proof. We follow Protter [11] rather closely in his proof of the Itô formula for general semimartingales. Let $\mathcal{P}_{n}$ be a refining sequence of Riemann partitions of $[0, T], \mathcal{P}_{n}=\{0=$ $\left.t_{0}^{n} \leq \cdots \leq t_{l_{n}}^{n}=T\right\}$

$$
C_{t}^{\mathcal{P}_{n}}(\Phi)=\sum_{k=1}^{n} f^{\prime}\left(\beta_{t_{k}}-\beta_{t_{k-1}}+\left(\delta_{t_{k}}-\delta_{t_{k-1}}\right) S_{t_{k}}\right)
$$

Since $\beta, \delta$, and $S$ are càdlàg processes, and $\sum_{s}\left(\Delta \beta_{s}\right)^{2}, \sum_{s}\left(\Delta \delta_{s}\right)^{2}$, and $\sum_{s}\left(\Delta S_{s}\right)^{2}$ are (absolutely) convergent series, given $\epsilon>0$, we can find two sets $A$ and $B$ such that $A$ and $B$ are disjoint and $A \cup B$ exhausts the jump times of $\beta, \delta$, and $S$ on $(0, T], A$ being a set of jump times that $\beta, \delta$, and $S$ have a.s. a finite number of times and $B$ being such that $\sum_{0<s \leq t}(\Delta \beta)^{2} \leq \epsilon^{2}, \sum_{0<s \leq t}(\Delta \delta)^{2} \leq \epsilon^{2}$, and $\sum_{0<s \leq t}(\Delta S)^{2} \leq \epsilon^{2}$.

Thus we have

$$
C_{t}^{\mathcal{P}}(\Phi)=\sum_{k, A} f^{\prime}\left(\beta_{t_{k}}-\beta_{t_{k-1}}+\left(\delta_{t_{k}}-\delta_{t_{k-1}}\right) S_{t_{k}}\right)+\sum_{k, B} f^{\prime}\left(\beta_{t_{k}}-\beta_{t_{k-1}}+\left(\delta_{t_{k}}-\delta_{t_{k-1}}\right) S_{t_{k}}\right)
$$

where $\sum_{k, A}$ denotes $\sum_{k} 1_{\left\{A \cap\left(t_{k-1}, t_{k}\right] \neq \emptyset\right\}}$ and $\sum_{k, B}$ denotes $\sum_{k} 1_{\left\{B \cap\left(t_{k-1}, t_{k}\right] \neq \emptyset\right\}}$.

The first sum converges to $\sum_{s \in A} f^{\prime}\left(\Delta \beta_{s}+\Delta \delta_{s} S_{s}\right)$.

In the second sum we apply Taylor's theorem, which says

$$
f^{\prime}(x)=f^{\prime \prime}(0) x+\frac{1}{2} f^{(3)}(0) x^{2}+R(x)
$$

where $|R(x)| \leq r(x) x^{2}$ such that $r: \mathbb{R}_{+} \rightarrow \mathbb{R}_{+}$is an increasing function with $\lim _{u \downarrow 0} r(u)=0$.

\footnotetext{
${ }^{4}$ The authors came across a similar result in the work of Diop [4].
} 
Thus we have

$$
\begin{aligned}
& \sum_{k, B} f^{\prime}\left(\beta_{t_{k}}-\beta_{t_{k-1}}+\left(\delta_{t_{k}}-\delta_{t_{k-1}}\right) S_{t_{k}}\right) \\
& =f^{\prime \prime}(0) \sum_{k, B}\left(\beta_{t_{k}}-\beta_{t_{k-1}}+\left(\delta_{t_{k}}-\delta_{t_{k-1}}\right) S_{t_{k}}\right) \\
& \quad+\frac{1}{2} f^{(3)}(0) \sum_{k, B}\left(\beta_{t_{k}}-\beta_{t_{k-1}}+\left(\delta_{t_{k}}-\delta_{t_{k-1}}\right) S_{t_{k}}\right)^{2} \\
& \quad+\sum_{k, B} R\left(\beta_{t_{k}}-\beta_{t_{k-1}}+\left(\delta_{t_{k}}-\delta_{t_{k-1}}\right) S_{t_{k}}\right) .
\end{aligned}
$$

The first sum (3) is equal to

$$
\sum_{k}\left(\beta_{t_{k}}-\beta_{t_{k-1}}+\left(\delta_{t_{k}}-\delta_{t_{k-1}}\right) S_{t_{k}}\right)-\sum_{k \in A}\left(\beta_{t_{k}}-\beta_{t_{k-1}}+\left(\delta_{t_{k}}-\delta_{t_{k-1}}\right) S_{t_{k}}\right),
$$

which converges in ucp topology to

$$
\left(V_{t}-V_{0}-\int_{0+}^{t} \delta_{s-} d S_{s}\right)-\sum_{s \in A}\left(\Delta \beta_{s}+\Delta \delta_{s} S_{s}\right) .
$$

The second sum (4), after developing and switching to less cumbersome notations, is equal to

$$
\sum_{k, B}\left(\beta_{k}-\beta_{k-1}\right)^{2}+2 S_{k}\left(\beta_{k}-\beta_{k-1}\right)\left(\delta_{k}-\delta_{k-1}\right)+S_{k}^{2}\left(\delta_{k}-\delta_{k-1}\right)^{2} .
$$

$\sum_{k, B}\left(\beta_{k}-\beta_{k-1}\right)^{2}=\sum_{k}\left(\beta_{k}-\beta_{k-1}\right)^{2}-\sum_{k, A}\left(\beta_{k}-\beta_{k-1}\right)^{2}$, and the first sum converges to $[\beta, \beta]_{t}$ while $\sum_{k \in A}\left(\beta_{k}-\beta_{k-1}\right)^{2}$ converges to $\sum_{s \in A} \Delta \beta_{s}^{2}$.

Now $\sum_{k, B} 2 S_{k}\left(\beta_{k}-\beta_{k-1}\right)\left(\delta_{k}-\delta_{k-1}\right)=\sum_{k, B} 2 S_{k-1}\left(\beta_{k}-\beta_{k-1}\right)\left(\delta_{k}-\delta_{k-1}\right)+\sum_{k, B}\left(S_{k}-\right.$ $\left.S_{k-1}\right)\left(\beta_{k}-\beta_{k-1}\right)\left(\delta_{k}-\delta_{k-1}\right)$.

The first term is equal to $\sum_{k} 2 S_{k-1}\left(\beta_{k}-\beta_{k-1}\right)\left(\delta_{k}-\delta_{k-1}\right)-\sum_{k, A} 2 S_{k-1}\left(\beta_{k}-\beta_{k-1}\right)\left(\delta_{k}-\delta_{k-1}\right)$ and converges to $2 \int_{0+}^{t} S_{s-} d[\beta, \delta]_{s}-2 \sum_{s \in A} S_{s-} \Delta \beta_{s} \Delta \delta_{s}$.

The second term is less than $\sup _{k, B}\left|\left(S_{k}-S_{k-1}\right)\right| \sum_{k, B}\left|\beta_{k}-\beta_{k-1}\right|\left|\delta_{k}-\delta_{k-1}\right|$ and again less than $\sup _{k, B}\left|\left(S_{k}-S_{k-1}\right)\right|\left(\sum_{k}\left(\beta_{k}-\beta_{k-1}\right)^{2}+\sum_{k}\left(\delta_{k}-\delta_{k-1}\right)^{2}\right.$. Taking the limit when $n \rightarrow \infty$ we find that $\left|\sum_{k, B}\left(S_{k}-S_{k-1}\right)\left(\beta_{k}-\beta_{k-1}\right)\left(\delta_{k}-\delta_{k-1}\right)\right| \leq \epsilon \sqrt{[\delta, \delta]_{t}} \sqrt{[\beta, \beta]_{t}}$.

Finally

$$
\begin{aligned}
\sum_{k, B} S_{k}^{2}\left(\delta_{k}-\delta_{k-1}\right)^{2}= & \sum_{k, B} S_{k-1}^{2}\left(\delta_{k}-\delta_{k-1}\right)^{2} \\
& +2 \sum_{k, B} S_{k-1}\left(S_{k}-S_{k-1}\right)\left(\delta_{k}-\delta_{k-1}\right)^{2}+\sum_{k, B}\left(S_{k}-S_{k-1}\right)^{2}\left(\delta_{k}-\delta_{k-1}\right)^{2} .
\end{aligned}
$$

The first term is equal to $\sum_{k} S_{k-1}^{2}\left(\delta_{k}-\delta_{k-1}\right)^{2}-\sum_{k, A} S_{k-1}^{2}\left(\delta_{k}-\delta_{k-1}\right)^{2}$ and converges to $\int_{0+}^{t} S_{s-}^{2} d[\delta, \delta]_{s}-\sum_{s \in A} S_{s-}^{2}\left(\Delta \delta_{s}\right)^{2}$. 
The second term is less than $\sup _{k, B}\left|S_{k}\right| \sup _{k, B}\left|\left(S_{k}-S_{k-1}\right)\right|\left(\sum_{k}\left(\delta_{k}-\delta_{k-1}\right)^{2}\right.$, and if we assume for now that $S \leq K<\infty$ uniformly in $t$, then we have $\mid \sum_{k, B} S_{k-1}\left(S_{k}-S_{k-1}\right)\left(\delta_{k}-\right.$ $\left.\delta_{k-1}\right)^{2} \mid \leq K \epsilon[\delta, \delta]_{t}$.

The last term is less than $\epsilon^{2}[\delta, \delta]_{t}$ by following the same reasoning.

Now we turn to the last term (5) of Taylor's development:

$$
\begin{aligned}
& \left|\sum_{k, B} R\left(\beta_{t_{k}}-\beta_{t_{k-1}}+\left(\delta_{t_{k}}-\delta_{t_{k-1}}\right) S_{t_{k}}\right)\right| \\
& \quad \leq \sum_{k, B} r\left(\left|\beta_{t_{k}}-\beta_{t_{k-1}}+\left(\delta_{t_{k}}-\delta_{t_{k-1}}\right) S_{t_{k}}\right|\right)\left(\beta_{t_{k}}-\beta_{t_{k-1}}+\left(\delta_{t_{k}}-\delta_{t_{k-1}}\right) S_{t_{k}}\right)^{2}
\end{aligned}
$$

Again, assuming that $\sup S \leq K \leq \infty$ over $[0, T]$, we have $(6) \leq \sup r((K+1) \epsilon)[\delta, \delta]_{t}$.

We are now ready to take the limit when $\epsilon$ goes to zero. The last term tends to zero from the property of $r$, and it remains to prove that the series $\sum_{s \in A}$ are absolutely convergent. We next proceed by localization, as in Protter [11], by considering first $V_{K}=\inf \{t>0,|\delta|>K\}$, $W_{K}=\inf \{t>0,|\beta|>K\}$, and $Z_{K}=\inf \{t>0,|S|>K\}$ so that $1_{\left[0, V_{K}\right]} \delta, 1_{\left[0, W_{K}\right]} \beta$, and $1_{\left[0, Z_{K}\right]} S$ are $[-K, K]$-valued. Therefore we have that $\left|f^{\prime}(x)-f^{\prime \prime}(0) x\right| \leq C x^{2}$ for some constant $C$. This allows us to write

$$
\begin{gathered}
\left|\sum_{s \in A} f^{\prime}\left(\Delta \beta_{s}+\Delta \delta S_{s}\right)-f^{\prime \prime}(0)\left(\sum_{s \in A} \Delta \beta_{s}+\Delta \delta_{s} S_{s}\right)\right| \\
\leq C \sum_{s \in A} \Delta \beta_{s}^{2}+2 \Delta \beta_{s} \Delta \delta S_{s}+\Delta \delta_{s}^{2} S_{s}^{2} \\
\leq C\left([\beta, \beta]_{t}+2 K\left|[\delta, \beta]_{t}\right|+K^{2}[\delta, \delta]_{t}\right)<\infty .
\end{gathered}
$$

And the series are absolutely convergent, which completes the proof.

Corollary 1. The $f$-cost process of an $H$-admissible strategy $\Phi$ can also be expressed in terms of the portfolio value $V$ :

$$
\begin{aligned}
C_{t}(\Phi)= & f^{\prime \prime}(0)\left(V_{t}-V_{0}-\int_{0+}^{t} \delta_{s-} d S_{s}\right) \\
& +\frac{f^{(3)}(0)}{2}\left([V, V]_{t}^{c}-2 \int_{0+}^{t} \delta_{s-} d[V, S]_{s}^{c}+\int_{0+}^{t} \delta_{s-}^{2} d[S, S]_{s}^{c}\right) \\
& +\sum_{0<s \leq t} f^{\prime}\left(\Delta V_{s}-\delta_{s-} \Delta S_{s}\right)-f^{\prime \prime}(0)\left(\Delta V_{s}-\delta_{s-} \Delta S_{s}\right) .
\end{aligned}
$$

Proof. The proof follows easily from applying the definition of $V$ in the formula (2) and straightforward calculations using quadratic variation properties.

With the $f$-cost process well defined for strategies of interest in continuous time, we can now state the criteria which will characterize pseudo-optimal strategies, by analogy with the discrete time case.

Copyright $\odot$ by SIAM. Unauthorized reproduction of this article is prohibited. 
Definition. An $H$-admissible strategy $\Phi$ will be called pseudo-optimal for the local riskminimization if its $f$-cost process is a martingale strongly orthogonal to the martingale part $M$ of the process $S$.

In the next section, we will derive the corresponding set of equations that pseudo-optimal strategies have to solve in two different Markovian frameworks.

4. Application to stochastic volatility models. In this section we will assume further hypotheses on the trading strategies so that we can derive an explicit formula for the $f$ cost process and completely characterize the pseudo-optimal strategies for the local riskminimization.

We model the evolution of $S$ through a set of SDEs with stochastic volatility:

$$
\begin{aligned}
d S_{t} & =\mu_{t} d t+\sigma_{t} d W_{t}^{1}, \\
d \sigma_{t} & =\gamma_{t} d t+\Sigma_{t} d W_{t}^{2}
\end{aligned}
$$

with smooth $\mu_{t}, \gamma_{t}$, and $\Sigma_{t}, W^{1}$ and $W^{2}$ being standard Wiener processes under $\mathcal{P}$ with constant instantaneous correlation $\rho$, i.e., $d\left\langle W^{1}, W^{2}\right\rangle_{t}=\rho d t$.

We shall also assume that appropriate conditions hold for the functions $\mu_{t}, \gamma_{t}$, and $\Sigma_{t}$ so that the system of SDEs (8), (9) admits a unique strong continuous solution for $S$ and $\sigma$, with $S>0$ and $\sigma>0$. With these diffusion assumptions we will now place ourselves in a Markovian framework and look for the optimal strategy $\Phi$ as a smooth function of the state variables

$$
\begin{aligned}
\delta_{t} & =\delta\left(t, S_{t}, \sigma_{t}\right), \\
V_{t} & =V\left(t, S_{t}, \sigma_{t}\right) .
\end{aligned}
$$

4.1. PDE formulation. We first derive a PDE formulation. For that purpose let us express the cost process as a function of the diffusion parameters and the strategy

$$
\begin{aligned}
C_{t}(\Phi)=\int_{0}^{t}\left[f^{\prime \prime}(0)\left(\frac{\partial V}{\partial u}+\frac{\partial V}{\partial S} \mu_{u}+\frac{\partial V}{\partial \sigma} \gamma_{u}+\frac{1}{2} \frac{\partial^{2} V}{\partial S^{2}} \sigma_{u}^{2}+\frac{1}{2} \frac{\partial^{2} V}{\partial \sigma^{2}} \Sigma_{u}^{2}+\frac{\partial^{2} V}{\partial S \partial \sigma} \rho \sigma_{u} \Sigma_{u}-\delta_{u} \mu_{u}\right)\right. \\
+\frac{f^{(3)}(0)}{2}\left(\left(\frac{\partial V}{\partial S}\right)^{2} \sigma_{u}^{2}+\left(\frac{\partial V}{\partial \sigma}\right)^{2} \Sigma_{u}^{2}+2 \frac{\partial V}{\partial S} \frac{\partial V}{\partial \sigma} \rho \sigma_{u} \Sigma_{u}\right) \\
\left.\quad-f^{(3)}(0) \delta_{u}\left(\frac{\partial V}{\partial S} \sigma_{u}^{2}+\frac{\partial V}{\partial \sigma} \rho \sigma_{u} \Sigma_{u}\right)+\frac{f^{(3)}(0)}{2} \delta_{u}^{2} \sigma_{u}^{2}\right] d u \\
+\int_{0}^{t} f^{\prime \prime}(0)\left(\frac{\partial V}{\partial S}-\delta_{u}\right) \sigma_{u} d W_{u}^{1}+\int_{0}^{t} f^{\prime \prime}(0) \frac{\partial V}{\partial \sigma} \Sigma_{u} d W_{u}^{2}
\end{aligned}
$$

which we have obtained from (7).

Copyright ( ) by SIAM. Unauthorized reproduction of this article is prohibited. 
Now, applying to the strategy $\Phi$ the first pseudo-optimality criterion, i.e., that $C$ must be a martingale under the measure $P$, we find the PDE satisfied by the portfolio value $V$ :

$$
\begin{aligned}
& f^{\prime \prime}(0)\left(\frac{\partial V}{\partial u}+\frac{\partial V}{\partial S} \mu_{u}+\right.\left.\frac{\partial V}{\partial \sigma} \gamma_{u}+\frac{1}{2} \frac{\partial^{2} V}{\partial S^{2}} \sigma_{u}^{2}+\frac{1}{2} \frac{\partial^{2} V}{\partial \sigma^{2}} \Sigma_{u}^{2}+\frac{\partial^{2} V}{\partial S \partial \sigma} \rho \sigma_{u} \Sigma_{u}-\delta_{u} \mu_{u}\right) \\
&+\frac{f^{(3)}(0)}{2}\left(\left(\frac{\partial V}{\partial S}\right)^{2} \sigma_{u}^{2}+\left(\frac{\partial V}{\partial \sigma}\right)^{2} \Sigma_{u}^{2}+2 \frac{\partial V}{\partial S} \frac{\partial V}{\partial \sigma} \rho \sigma_{u} \Sigma_{u}\right) \\
&-f^{(3)}(0) \delta_{u}\left(\frac{\partial V}{\partial S} \sigma_{u}^{2}+\frac{\partial V}{\partial \sigma} \rho \sigma_{u} \Sigma_{u}\right)+\frac{f^{(3)}(0)}{2} \delta_{u}^{2} \sigma_{u}^{2}=0
\end{aligned}
$$

with the terminal conditions corresponding to $V_{T}=\delta^{H} S_{T}+\beta^{H}$.

Applying to the strategy $\Phi$ the second pseudo-optimality criterion, i.e., that the martingale $C$ must be orthogonal to $S$, we find the equation satisfied by the optimal hedge $\delta$ :

$$
\left(\frac{\partial V}{\partial S}-\delta_{u}\right) \sigma_{u}^{2}+\frac{\partial V}{\partial \sigma} \rho \sigma_{u} \Sigma_{u}=0
$$

Complete markets case. The case of complete markets allows us to recover the celebrated Black and Scholes formula [3], [10] regardless of the choice for the function $f$. Indeed, by taking $\Sigma$ the volatility of volatility equal to zero, the optimality equations reduce to

$$
\begin{aligned}
\delta_{u} & =\frac{\partial V}{\partial S}, \\
\frac{\partial V}{\partial u}+\frac{1}{2} \frac{\partial^{2} V}{\partial S^{2}} \sigma_{u}^{2} & =0 .
\end{aligned}
$$

Equation (10) gives the perfect hedging strategy in that context, since upon suitable boundary conditions it is well known that the PDE (11) has a unique solution. Of course one can verify that the $f$-cost process is then identically zero, which amounts to having a self-financing strategy that perfectly replicates the contingent claim $H$.

4.2. Link with FBSDE. Given the two equations we found for the optimal portfolio, we can now relate the pseudo-optimal strategies for the local risk-minimization with the solution of an FBSDE associated with the diffusion process of the stock price $S$. This is based on the generalization of the Feynman-Kac formula (see the survey paper on BSDEs in finance from El Karoui, Peng and Quenez [5], for instance), which links quasi-linear PDEs with BSDEs.

We begin by rewriting the two equations satisfied by the theoretical portfolio value $V_{t}$ and the stock quantity $\delta_{t}$ as

$$
\begin{aligned}
& \frac{\partial V}{\partial u}+\frac{\partial V}{\partial S} \mu_{u}+\frac{\partial V}{\partial \sigma} \gamma_{u}+\frac{1}{2} \frac{\partial^{2} V}{\partial S^{2}} \sigma_{u}^{2}+\frac{1}{2} \frac{\partial^{2} V}{\partial \sigma^{2}} \Sigma_{u}^{2}+\frac{\partial^{2} V}{\partial S \partial \sigma} \rho \sigma_{u} \Sigma_{u} \\
& \quad=\delta_{u} \mu_{u}+\alpha\left(\left(\frac{\partial V}{\partial S} \sigma_{u}+\frac{\partial V}{\partial \sigma} \rho \Sigma_{u}-\delta_{u} \sigma_{u}\right)^{2}+\left(1-\rho^{2}\right)\left(\frac{\partial V}{\partial \sigma}\right)^{2} \Sigma^{2}\right) \\
& \frac{\partial V}{\partial S} \sigma_{u}+\frac{\partial V}{\partial \sigma} \rho \Sigma_{u}-\delta_{u} \sigma_{u}=0
\end{aligned}
$$

Copyright (C) by SIAM. Unauthorized reproduction of this article is prohibited. 
where $\alpha=-\frac{1}{2} \frac{f^{(3)}(0)}{f^{\prime \prime}(0)}$. Inserting (13) in (12), we find

$$
\begin{aligned}
\frac{\partial V}{\partial u}+\Lambda V & =\frac{\mu}{\sigma}\left(\frac{\partial V}{\partial S} \sigma+\frac{\partial V}{\partial \sigma} \rho \Sigma\right)+\alpha\left(\sqrt{1-\rho^{2}} \frac{\partial V}{\partial \sigma} \Sigma\right)^{2}, \\
\delta \sigma & =\frac{\partial V}{\partial S} \sigma+\frac{\partial V}{\partial \sigma} \rho \Sigma,
\end{aligned}
$$

where $\Lambda$ is the infinitesimal generator corresponding to the diffusion equations (8) and (9) under the measure $\mathcal{P}$ :

$$
\Lambda V_{u}=\frac{\partial V}{\partial S} \mu_{u}+\frac{\partial V}{\partial \sigma} \gamma_{u}+\frac{1}{2} \frac{\partial^{2} V}{\partial S^{2}} \sigma_{u}^{2}+\frac{1}{2} \frac{\partial^{2} V}{\partial \sigma^{2}} \Sigma_{u}^{2}+\frac{\partial^{2} V}{\partial S \partial \sigma} \rho \sigma_{u} \Sigma_{u}
$$

So we are now in a position to state the main result of this section, effectively relating a pseudo-optimal strategy with the solution of an FBSDE.

Theorem 3. Any pseudo-optimal strategy $\Phi=(V, \delta)$ for the local risk-minimization yields a solution to the following FBSDE:

$$
\begin{aligned}
d S_{t} & =\mu_{t} d t+\sigma_{t} d W_{t}^{1} \\
d \sigma_{t} & =\gamma_{t} d t+\Sigma_{t}\left(\rho d W_{t}^{1}+\sqrt{1-\rho^{2}} d W_{t}^{2}\right), \\
-d Y_{s} & =g\left(s, S_{s}, \sigma_{s}, Y_{s}, Z_{s}^{1}, Z_{s}^{2}\right) d s-Z_{s}^{1} d W_{s}^{1}-Z_{s}^{2} d W_{s}^{2}, \\
Y_{T} & =\beta^{H}+\delta^{H} S_{T},
\end{aligned}
$$

where $W=\left(W^{1}, W^{2}\right)$ is a standard two-dimensional Brownian motion and $g(s, S, \sigma, Y$, $\left.Z^{1}, Z^{2}\right)=-\frac{\mu}{\sigma} Z^{1}-\alpha\left(Z^{2}\right)^{2}$, with $Y=V$ and $Z=\left(\delta \sigma, \frac{\partial V}{\partial \sigma} \Sigma \sqrt{1-\rho^{2}}\right)$.

Proof. The result follows from application of the Itô formula to the pseudo-optimal strategy $\Phi^{*}=\left(V^{*}, \delta^{*}\right)$, which then solve (14) and (15). We get

$$
\begin{aligned}
& d V_{t}^{*}=\frac{\partial V}{\partial t}+\Lambda V_{t}+\frac{\partial V}{\partial S} \sigma d W_{s}^{1}+\frac{\partial V}{\partial \sigma} \Sigma\left(\rho d W_{s}^{1}+\sqrt{1-\rho^{2}} d W_{s}^{2}\right) \\
& \Leftrightarrow d V_{t}^{*}=-g\left(t, S_{t}, \sigma_{t}, V_{t}, \delta_{t}^{*}, \sqrt{1-\rho^{2}} \frac{\partial V}{\partial \sigma} \Sigma\right)+\frac{\partial V}{\partial S} \sigma d W_{s}^{1}+\frac{\partial V}{\partial \sigma} \Sigma\left(\rho d W_{s}^{1}+\sqrt{1-\rho^{2}} d W_{s}^{2}\right) \\
& \Leftrightarrow-d V_{t}^{*}=g\left(t, S_{t}, \sigma_{t}, V_{t}, \delta_{t}^{*}, \sqrt{1-\rho^{2}} \frac{\partial V}{\partial \sigma} \Sigma\right)-\delta^{*} \sigma_{s} d W_{s}^{1}-\frac{\partial V}{\partial \sigma} \Sigma \sqrt{1-\rho^{2}} d W_{s}^{2},
\end{aligned}
$$

which is the result announced with $Y=V$ and $Z=\left(\delta \sigma, \frac{\partial V}{\partial \sigma} \Sigma \sqrt{1-\rho^{2}}\right)$.

4.3. The minimization problem. After using the pseudo-optimal criteria to characterize strategies, we return to the original minimization problem to show that those identified strategies are actually optimal.

Given the smoothness of the risk function $f$, we can rewrite the process $r_{f}^{\tau}$ by using a Taylor development around the nonperturbed strategy $\Phi$. Let us fix $t \in[0, T]$; because of the definition of the process $r_{f}^{\tau}[\Phi, \phi]$ and as we work with increasing sequences of partitions, we may assume that $t$ is one of the $t_{i(n)}^{n}$ (we will thereafter drop the superscript $n$ and simply 
write $t_{i}$ instead), and we have

$$
\begin{aligned}
r_{f}^{\tau}[\Phi, \phi](t, \omega) & =\frac{\Delta R_{t_{i}}\left(\Phi+\left.\phi\right|_{\left[t_{i}, t_{i+1}(\right.}\right)(\omega)-\Delta R_{t_{i}}(\Phi)(\omega)}{t_{i+1}-t_{i}} \\
& =\frac{\mathbb{E}_{t_{i}}\left(f\left(\Delta C_{t_{i+1}}\left(\Phi+\left.\phi\right|_{\left[t_{i}, t_{i+1}\right.}()\right)\right)(\omega)-\mathbb{E}_{t_{i}}\left(f\left(\Delta C_{t_{i+1}}(\Phi)\right)\right)(\omega)\right.}{t_{i+1}-t_{i}} .
\end{aligned}
$$

Applying Taylor's formula with remainder term to $g:(x, y) \mapsto f(x+y)$ in the expectation, we have that

$$
\begin{aligned}
f\left(\Delta C_{t_{i+1}}\left(\Phi+\left.\phi\right|_{\left[t_{i}, t_{i+1}()\right.}\right)\right)=f\left(\Delta C_{t_{i+1}}(\Phi)\right) & -\beta_{t_{i}} f^{\prime}\left(\Delta C_{t_{i+1}}(\Phi)\right) \\
& -\delta_{t_{i}} S_{t_{i+1}} f^{\prime}\left(\Delta C_{t_{i+1}}(\Phi)\right)+\frac{1}{2}\left(\beta_{t_{i}}+\delta_{t_{i}} S_{t_{i+1}}\right)^{2} g(\tilde{\phi}),
\end{aligned}
$$

where $g(\tilde{\phi})=f^{\prime \prime}(\Delta C(\tilde{\phi}))$ with $\tilde{\phi}=(\tilde{\beta}, \tilde{\delta})$ such that $|\tilde{\beta}| \leq \beta$ and $|\tilde{\delta}| \leq \delta$.

Rearranging and simplifying we get

$$
\begin{aligned}
r_{f}^{\tau}[\phi, \Delta](t, \omega)= & \beta_{t_{i}} \frac{\mathbb{E}_{t_{i}}\left(f^{\prime}\left(\Delta C_{t_{i+1}}(\phi)\right)\right)(\omega)}{t_{i+1}-t_{i}}+\delta_{t_{i}} \frac{\mathbb{E}_{t_{i}}\left(S_{t_{i+1}} f^{\prime}\left(\Delta C_{t_{i+1}}(\phi)\right)\right)(\omega)}{t_{i+1}-t_{i}} \\
& +\frac{\mathbb{E}_{t_{i}}\left(\left(\beta_{t_{i}}+\frac{1}{2} \delta_{t_{i}} S_{t_{i+1}}\right)^{2} g(\tilde{\phi})\right)(\omega)}{t_{i+1}-t_{i}} .
\end{aligned}
$$

Because we work with Itô processes, the following stand:

$$
\begin{aligned}
\lim _{t_{i+1} \rightarrow t_{i}} \frac{\mathbb{E}_{t_{i}}\left(f^{\prime}\left(\Delta C_{t_{i+1}}(\phi)\right)\right)(\omega)}{t_{i+1}-t_{i}} & =\Lambda\left(f^{\prime} \circ \Delta\right)_{t_{i}}, \\
\lim _{t_{i+1} \rightarrow t_{i}} \frac{\mathbb{E}_{t_{i}}\left(S_{t_{i+1}} f^{\prime}\left(\Delta C_{t_{i+1}}(\phi)\right)\right)(\omega)}{t_{i+1}-t_{i}} & =\Lambda\left(S \cdot f^{\prime} \circ \Delta\right)_{t_{i}}
\end{aligned}
$$

and

$$
\begin{aligned}
\lim _{t_{i+1} \rightarrow t_{i}} \frac{\mathbb{E}_{t_{i}}(g(\tilde{\phi}))(\omega)}{t_{i+1}-t_{i}} & =\Lambda g_{t_{i}}, \\
\lim _{t_{i+1} \rightarrow t_{i}} \frac{\mathbb{E}_{t_{i}}\left(S_{t_{i+1}} g(\tilde{\phi})\right)(\omega)}{t_{i+1}-t_{i}} & =\Lambda(S \cdot g)_{t_{i}}, \\
\lim _{t_{i+1} \rightarrow t_{i}} \frac{\mathbb{E}_{t_{i}}\left(S_{t_{i+1}}^{2} g(\tilde{\phi})\right)(\omega)}{t_{i+1}-t_{i}} & =\Lambda\left(S^{2} \cdot g\right)_{t_{i}},
\end{aligned}
$$

where $\Lambda$ is the infinitesimal generator associated with the diffusion:

$$
\Lambda h=\frac{\partial h}{\partial S} \mu+\frac{\partial h}{\partial \sigma} \gamma+\frac{1}{2} \frac{\partial^{2} h}{\partial S^{2}} \sigma^{2}+\frac{1}{2} \frac{\partial^{2} h}{\partial \sigma^{2}} \Sigma^{2}+\frac{\partial^{2} h}{\partial S \partial \sigma} \rho \sigma \Sigma .
$$

Copyright (C by SIAM. Unauthorized reproduction of this article is prohibited. 
Finally the process $r_{f}^{\tau}$ evaluated in $t$ is worth

$$
\begin{aligned}
r_{f}^{\tau}[\phi, \Delta](t, \omega)= & \beta_{t} \Lambda\left(f^{\prime} \circ \Delta C\right)_{t}+\delta_{t} \Lambda\left(S \cdot f^{\prime} \circ \Delta C\right)_{t} \\
& +\frac{1}{2}\left(\beta_{t}^{2} \Lambda g_{t}+2 \beta_{t} \delta_{t} \Lambda(S \cdot g)_{t}+\delta_{t}^{2} \Lambda\left(S^{2} \cdot g\right)_{t}\right)
\end{aligned}
$$

Now we first take the component $\delta$ of the perturbation equal to zero, that is, we perturb only $\beta$, so that we have the following first condition for the strategy $\phi$ to be locally riskminimizing:

$$
\beta_{t} \Lambda\left(f^{\prime} \circ \Delta C\right)_{t}+\frac{1}{2} \beta_{t}^{2} \Lambda g_{t} \geq 0 \text { P-a.e. } \forall \beta_{t} .
$$

As a consequence we must have $\Lambda\left(f^{\prime} \circ \Delta C\right)_{t}=0$.

Likewise we take the component $\beta$ equal to zero, and we get the following second condition for the strategy $\phi$ to be locally risk-minimizing:

$$
\delta_{t} \Lambda\left(S \cdot f^{\prime} \circ \Delta C\right)_{t}+\frac{1}{2} \delta_{t}^{2} \Lambda\left(S^{2} \cdot g\right)_{t} \geq 0 \mathcal{P} \text {-a.e. } \quad \forall \delta_{t} .
$$

Therefore we must have $\Lambda\left(S \cdot f^{\prime} \circ \Delta C\right)_{t}=0$. But we observe that

$$
\left.\begin{array}{c}
\left\{\begin{array}{c}
\Lambda\left(f^{\prime} \circ \Delta C\right)_{t}=0, \\
\Lambda\left(S \cdot f^{\prime} \circ \Delta C\right)_{t}=0
\end{array}\right. \\
\Leftrightarrow
\end{array}\right\} \begin{gathered}
f^{\prime \prime}(0)\left(\frac{\partial V}{\partial t}+\frac{\partial V}{\partial S} \mu_{t}+\frac{\partial V}{\partial \sigma} \gamma_{t}+\frac{1}{2} \frac{\partial^{2} V}{\partial S^{2}} \sigma_{t}^{2}+\frac{1}{2} \frac{\partial^{2} V}{\partial \sigma^{2}} \Sigma_{t}^{2}+\frac{\partial^{2} V}{\partial S \partial \sigma} \rho \sigma_{t} \Sigma_{t}-\delta_{t} \mu_{t}\right), \\
+\frac{f^{(3)}(0)}{2}\left(\left(\frac{\partial V}{\partial S}\right)^{2} \sigma_{t}^{2}+\left(\frac{\partial V}{\partial \sigma}\right)^{2} \Sigma_{t}^{2}+2 \frac{\partial V}{\partial S} \frac{\partial V}{\partial \sigma} \rho \sigma_{t} \Sigma_{t}\right), \\
-f^{(3)}(0) \delta_{t}\left(\frac{\partial V}{\partial S} \sigma_{t}^{2}+\frac{\partial V}{\partial \sigma} \rho \sigma_{t} \Sigma_{t}\right)+\frac{f^{(3)}(0)}{2} \delta_{t}^{2} \sigma_{t}^{2}=0, \\
\left(\frac{\partial V}{\partial S}-\delta_{t}\right) \sigma_{t}^{2}+\frac{\partial V}{\partial \sigma} \rho \sigma_{t} \Sigma_{t}=0 .
\end{gathered}
$$

Finally, as announced in the beginning of this section, we see that in this context of stochastic volatility model, the optimal strategies with respect to the local risk-minimization problem are the same as the pseudo-optimal strategies. As one can see in the derivation, the only requirement to obtain this result is the existence of the infinitesimal generator and its expression in terms of the parameters of the SDE driving the process so as to identify the sets of two equations, which therefore allows for a straightforward generalization to Itô processes.

5. Application to stochastic volatility/jump diffusion models. In this section, we want to provide an example of a situation where the nonquadratic risk definitely implies a different hedging strategy, not only through the Taylor expansion around zero of the risk function. We therefore model the evolution of $S$ through an SDE with stochastic volatility and Poisson jumps in the vein of the Bates model [2]:

$$
\begin{aligned}
& d S_{t}=\mu_{t} d t+\sigma_{t} d W_{t}^{1}+k d N_{t}, \\
& d \sigma_{t}=\gamma_{t} d t+\Sigma_{t} d W_{t}^{2}
\end{aligned}
$$

Copyright ( $)$ by SIAM. Unauthorized reproduction of this article is prohibited. 
with $W^{1}$ and $W^{2}$ Wiener processes under $\mathcal{P}$ and $d\left\langle W^{1}, W^{2}\right\rangle_{t}=\rho d t, N_{t}$ a Poisson process of intensity $\lambda$, and the amplitude of the jumps $k$ having probability distribution $K$. We also assume that $W_{t}, N_{t}$, and $k$ are independent. As in the case of stochastic volatility we shall assume that appropriate conditions hold on the adapted processes $\mu, \sigma, \gamma$, and $\Sigma$ so that the set of SDEs has a unique strong solution.

With these assumptions we will again place ourselves in a Markovian framework and look for the optimal strategy $\Phi$ as a smooth function of the state variables

$$
\begin{aligned}
\delta_{t} & =\delta\left(t, S_{t}, \sigma_{t}\right), \\
V_{t} & =V\left(t, S_{t}, \sigma_{t}\right) .
\end{aligned}
$$

5.1. PIDE formulation. We first derive a PIDE formulation. For that purpose let us express the cost process as a function of the diffusion parameters and the strategy

$$
\begin{aligned}
C_{t}(\Phi)= & \int_{0}^{t}\left(f^{\prime \prime}(0)\left(\frac{\partial V}{\partial u}+\frac{\partial V}{\partial S} \mu_{u}+\frac{\partial V}{\partial \sigma} \gamma_{u}+\frac{1}{2} \frac{\partial^{2} V}{\partial S^{2}} \sigma_{u}^{2}+\frac{1}{2} \frac{\partial^{2} V}{\partial \sigma^{2}} \Sigma_{u}^{2}+\frac{\partial^{2} V}{\partial S \partial \sigma} \rho \sigma_{u} \Sigma_{u}-\delta_{u-} \mu_{u}\right)\right. \\
& \quad+\frac{f^{(3)}(0)}{2}\left(\left(\frac{\partial V}{\partial S}\right)^{2} \sigma_{u}^{2}+\left(\frac{\partial V}{\partial \sigma}\right)^{2} \Sigma_{u}^{2}+2 \frac{\partial V}{\partial S} \frac{\partial V}{\partial \sigma} \rho \sigma_{u} \Sigma_{u}\right) \\
& \left.\quad-f^{(3)}(0) \delta_{u-}\left(\frac{\partial V}{\partial S} \sigma_{u}^{2}+\frac{\partial V}{\partial \sigma} \rho \sigma_{u} \Sigma_{u}\right)+\frac{f^{(3)}(0)}{2} \delta_{u-}^{2} \sigma_{u}^{2}\right) d u \\
& +\int_{0}^{t} f^{\prime \prime}(0)\left(\frac{\partial V}{\partial S}-\delta_{u-}\right) \sigma_{u} d W_{u}^{1} \\
& +\int_{0}^{t} f^{\prime \prime}(0) \frac{\partial V}{\partial \sigma} \Sigma_{u} d W_{u}^{2} \\
& +\int_{0}^{t} \int_{\mathbb{R}} f^{\prime}\left(\Delta V_{u}-\delta_{u-} \Delta S_{u}\right) K(k) d k d N_{u}
\end{aligned}
$$

which we have again obtained from (7) and with $\Delta V_{u}$ the jump in $V$ when there is a jump $\Delta S_{u}$ of size $k$ on $S$ at time $u$ being equal to $V\left(u-, S_{u-}+k, \sigma_{u-}\right)-V\left(u-, S_{u-}, \sigma_{u-}\right)$.

Now, applying to the strategy $\Phi$ the first pseudo-optimality criterion, i.e., that $C$ must be a martingale under the measure $P$, we find the PIDE satisfied by the portfolio value $V$ :

$$
\begin{aligned}
& f^{\prime \prime}(0)\left(\frac{\partial V}{\partial u}+\frac{\partial V}{\partial S} \mu_{u}+\right.\left.\frac{\partial V}{\partial \sigma} \gamma_{u}+\frac{1}{2} \frac{\partial^{2} V}{\partial S^{2}} \sigma_{u}^{2}+\frac{1}{2} \frac{\partial^{2} V}{\partial \sigma^{2}} \Sigma_{u}^{2}+\frac{\partial^{2} V}{\partial S \partial \sigma} \rho \sigma_{u} \Sigma_{u}-\delta_{u-} \mu_{u}\right) \\
&+\frac{f^{(3)}(0)}{2}\left(\left(\frac{\partial V}{\partial S}\right)^{2} \sigma_{u}^{2}+\left(\frac{\partial V}{\partial \sigma}\right)^{2} \Sigma_{u}^{2}+2 \frac{\partial V}{\partial S} \frac{\partial V}{\partial \sigma} \rho \sigma_{u} \Sigma_{u}\right) \\
&-f^{(3)}(0) \delta_{u-}\left(\frac{\partial V}{\partial S} \sigma_{u}^{2}+\frac{\partial V}{\partial \sigma} \rho \sigma_{u} \Sigma_{u}\right)+\frac{f^{(3)}(0)}{2} \delta_{u-}^{2} \sigma_{u}^{2} \\
&+\int_{\mathbb{R}} f^{\prime}\left(\Delta V_{u}-\delta_{u-} \Delta S_{u}\right) K(k) d k \lambda_{u}=0
\end{aligned}
$$

with the terminal conditions corresponding to $V_{T}=\delta^{H} S_{T}+\beta^{H}$.

Copyright (c) by SIAM. Unauthorized reproduction of this article is prohibited. 
Applying to the strategy $\Phi$ the second pseudo-optimality criterion, i.e., that the martingale $C$ must be orthogonal to $S$, we find the equation satisfied by the optimal hedge $\delta$ :

$$
\left(\frac{\partial V}{\partial S}-\delta_{u-}\right) \sigma_{u}^{2}+\frac{\partial V}{\partial \sigma} \rho \sigma_{u} \Sigma_{u}+\int_{\mathbb{R}} f^{\prime}\left(\Delta V_{u}-\delta_{u-} \Delta S_{u}\right) k K(k) d k \lambda_{u}=0 .
$$

Contrarily to the stochastic volatility case, where only the local behavior of the risk function $f$ in 0 mattered, the optimal strategy in the jump-diffusion model requires the knowledge of the risk function $f$ on its whole support.

Conclusion. We have introduced in this article a new methodology in order to hedge contingent claims in incomplete markets. This methodology gives more flexibility in the choice of the function used to assess the risk of the strategy, and we believe it can fit better with market practice. Furthermore, being a local minimization of a local risk, it is expected to be more robust to fluctuations of market conditions so that traders will be able to adapt their hedging strategy at lower costs than with a more global approach. Finally, since the methodology defines the risk as a function of the trading costs, transaction costs can be included in the setting rather seamlessly. This will be shown in the case of liquidity costs in a forthcoming paper [1]. Further extensions include multidimensional semimartingales setting to describe the stock price processes and therefore deal with multiasset options.

\section{REFERENCES}

[1] F. Abergel And N. Millot, Non Quadratic Locally Risk-Minimizing Strategies: Incorporating Liquidity Costs, working paper.

[2] D. BATES, Jump and stochastic volatility: Exchange rates processes implicit in Deutsche Mark options, Rev. Financ. Stud., 9 (1996), pp. 69-107.

[3] F. Black And M. Scholes, The pricing of options and corporate liabilities, J. Polit. Econ., 81 (1973), pp. 637-659.

[4] A. Diop, Convergence of Some Random Functionals of Discretized Semimartingales, Bernoulli Society Math. Stat. Prob., to appear.

[5] N. El Karoui, S. Peng, And M. C. Quenez, Backward stochastic differential equations in finance, Math. Finance, 7 (1997), pp. 1-71.

[6] H. Follmer And M. Schweizer, Hedging by sequential regression: An introduction to the mathematics of option trading, ASTIN Bull., 18 (1989), pp. 147-160.

[7] H. Follmer And M. Schweizer, Hedging of contingent claims under incomplete information, in Applied Stochastic Analysis, Gordon and Breach, New York, 1991.

[8] H. Follmer and D. Sondermann, Hedging of nonredundant contingent claims, in Contributions to Mathematical Economics, W. Hildenbrand and A. Mas-Cohell, eds., North-Holland, Amsterdam, 1986, pp. 205-223.

[9] D. Heath, E. Platen, And M. Schweizer, A comparison of two quadratic approaches to hedging in incomplete markets, Math. Finance, 11 (2001), pp. 385-413.

[10] R. C. Merton, Theory of rational option pricing, Bell J. Econom. Management Sci., 4 (1973), pp. 141183.

[11] P. Protter, Stochastic Integration and Differential Equations 2nd ed., Springer-Verlag, Heidelberg, 2005.

[12] M. SchweIzer, Option hedging for semimartingales, Stochastic Process. Appl., 37 (1991), pp. 339-363.

Copyright (C) by SIAM. Unauthorized reproduction of this article is prohibited. 